\title{
Comparative analysis of the complete chloroplast genome among Prunus mume, P. armeniaca, and P. salicina
}

\author{
Song Xue ${ }^{1,2}$, Ting Shi ${ }^{1}$, Wenjie Luo ${ }^{1}$, Xiaopeng $\mathrm{Ni}^{1}$, Shahid lqbal ${ }^{1}{ }^{1}$, Zhaojun Ni ${ }^{1}$, Xiao Huang ${ }^{1}$, Dan Yao ${ }^{1}$,
} Zhijun Shen ${ }^{2}$ and Zhihong Gao'

\begin{abstract}
Prunus mume Sieb. et Zucc., P. armeniaca L., and P. salicina L. are economically important fruit trees in temperate regions. These species are taxonomically perplexing because of shared interspecific morphological traits and variation, which are mainly attributed to hybridization. The chloroplast is cytoplasmically inherited and often used for evolutionary studies. We sequenced the complete chloroplast genomes of $P$. mume, P. armeniaca, and $P$. salicina using Illumina sequencing followed by de novo assembly. The three chloroplast genomes exhibit a typical quadripartite structure with conserved genome arrangement, structure, and moderate divergence. The lengths of the genomes are $157,815,157,797$, and 157,916 bp, respectively. The length of the large single-copy region (LSC) region is $86,113,86,283$, and $86,122 \mathrm{bp}$, and the length of the SSC region is $18,916,18,734$, and 19,028 bp; the IR region is 26,393, 26,390, and $26,383 \mathrm{bp}$, respectively. Each of the three chloroplast genomes encodes 133 genes, including 94 protein-coding, 31 tRNA, and eight rRNA genes. Differential gene analysis for the three species revealed that trnY-ATA is a unique gene in P. armeniaca; in contrast, the gene trnl-TAT is only present in P. mume and P. salicina, though the position of the gene in these chloroplast genomes differs. Further comparative analysis of the complete chloroplast genome sequences revealed that the ORF genes and the sequences of linked regions rps 16 and $\operatorname{atpA}$, atpH and atpl, trnc-GCA and psbD, ycf3 and $a t p B$, and $r p L 32$ and $n d h D$ are significantly different and may be used as molecular markers in taxonomic studies. Phylogenetic evolution analysis of the three species suggests that $P$. mume has a closer genetic relationship to P. armeniaca than to $P$. salicina.
\end{abstract}

\section{Introduction}

The evolutionary process occurring in stone fruit trees is an interesting topic. However, phylogenetic relationships among $P$. mume, $P$. armeniaca, and $P$. salicina have been problematic because of frequent hybridization, apomixis, presumed rapid radiation, and complex historical diversity. Genome sequencing is frequently used to analyze phylogenetic relationships, genetic diversity, and evolutionary studies $^{1}$. Three independent genomes

\footnotetext{
Correspondence: Zhihong Gao (gaozhihong@njau.edu.cn)

'College of Horticulture, Nanjing Agricultural University, 210095 Nanjing, China

2Jiangsu Key Laboratory for Horticultural Crop Genetic Improvement, Nanjing, China
}

These authors contributed equally: Song Xue, Ting Shi offering genetic information are those of the chloroplast, mitochondrion, and nucleus. Compared with the nuclear genome, the chloroplast genome has a small size, singleparental inheritance, low nucleotide substitution rate, haploid nature, and highly conserved genomic structure $^{2,3}$. Therefore, the chloroplast genome has been considered the perfect model for diversity and evolution studies.

The development of the chloroplast in plants is proposed to have initiated from multiple endosymbiosis of cyanobacteria and photosynthesis vectors ${ }^{4}$. The chloroplast is an organelle that exists in the cytoplasmic matrix and is enveloped by a bilayer membrane, with a flat ellipsoidal or spherical shape. In addition to

\section{(c) The Author(s) 2019}

(c) (i) Open Access This article is licensed under a Creative Commons Attribution 4.0 International License, which permits use, sharing, adaptation, distribution and reproduction in any medium or format, as long as you give appropriate credit to the original author(s) and the source, provide a link to the Creative Commons license, and indicate if changes were made. The images or other third party material in this article are included in the article's Creative Commons license, unless indicated otherwise in a credit line to the material. If material is not included in the article's Creative Commons license and your intended use is not permitted by statutory regulation or exceeds the permitted use, you will need to obtain permission directly from the copyright holder. To view a copy of this license, visit http://creativecommons.org/licenses/by/4.0/. 
photosynthesis, chloroplasts are involved in the synthesis of starch, fatty acids, pigments, and amino acids. Chloroplasts are also semi-autonomous genetic organelles and contain independent chloroplast DNA (cpDNA). The first chloroplast genome sequencing of tobacco was completed in $1986^{5}$, and at the end of March 2018, there were 13,602 complete plant chloroplast genomes collected at the National Center for Biotechnology Information (NCBI). In general, chloroplast DNA has a double-stranded, circular, typically four-segment structure, with a few linear molecular structures. It contains a LSC, a small singlecopy region (SSC), and a pair of reverse complementary repeat regions (IRs), with the IR region separating the LSC and SSC regions. The length of the genome is $\sim 120-160 \mathrm{~kb}^{6}$, and differences are mostly due to IR expansion/contraction or loss ${ }^{7,8}$. For example, the chloroplast genome of some algae does not contain an IR region $^{9,10}$. Some leguminous plants lose one of the IR regions ${ }^{10}$, whereas the chloroplast genome of Pisum sativum has lost the IR segment, which shortened its length. The chloroplast genome generally encodes 110-130 genes, which are highly conserved with regard to composition and sequence. Furthermore, the conserved structure of cpDNA and its low nucleotide substitution rate play a vital role in phylogenetic studies ${ }^{11}$.

The Rosaceae family contains over 120 genera and 3300 species with a great economic importance widely distributed in temperate regions ${ }^{12}$. The family can be divided into four subfamilies according to fruit type: Rosoideae, Prunoideae, Spiraeoideae, and Maloideae, with $P$. mume, $P$. armeniaca, and $P$. salicina belonging to Prunoideae. Nuclear genome sequences have been published for Rosa and Malus $\times$ domestica ${ }^{13}$, seven species of the genus Firago ${ }^{14}$, and Rubus occidentalis ${ }^{15}$, providing valuable information for evolutionary classification. However, due to apomixis, hybridization, and hypothetical rapid radiation, the phylogenetic relationship among Rosaceae species is complex ${ }^{12}$. The chloroplast genome of $P$. mume, $P$. armeniaca, and $P$. salicina can be used to understand the structure and rapid evolution of the Prunus genome, which will also help to illustrate and assess chloroplast genetic diversity. A profound analysis of phylogenetic relationships in Prunus species through chloroplast genome sequences would be valuable and interesting.

In this study, we completely sequences the chloroplast genomes of $P$. mume, $P$. armeniaca, and $P$. salicina using Illumina technology followed by reference-guided assembly of de novo contigs. Furthermore, we compared the chloroplast genomes of these three species with the complete chloroplast sequence of 23 other Rosaceae species and constructed a phylogenetic tree, which was further used for exploring genetic relationships among $P$. mume, $P$. armeniaca, and $P$. salicina using the entire chloroplast genome, coding regions, LSCs, IRs, and introns.

\section{Results}

Characterization of chloroplast genomes in Prunus species

A total of $27.49 \mathrm{~Gb}$ clean data were obtained after sequencing; regarding statistical assessment of base quality, we obtained $94.17 \%$ of Q30 bases. For P. mume, $P$. armeniaca, and P. salicina, 1,107,094, 662,524, and 824,216 paired-end reads and 297, 296, and $298 \mathrm{bp}$, respectively, of average insert size were produced by Illumina sequencing. The average organelle coverage for $P$. mume, $P$. armeniaca, and $P$. salicina with the reference genome reached 1059,634 , and 788 , respectively. The chloroplast genomes of the three Prunus species along with their size, reads, GC contents, and average are shown in Table 1. The entire genome size of Prunus species is similar to the reference peach genome, at $\sim 160 \mathrm{~kb}$. We obtained complete chloroplast genome maps of $P$. mume, $P$. armeniaca, and $P$. salicina through de novo genome sequencing and assembly (Fig. 1).

The chloroplast genomes of $P$. armeniaca, $P$. mume, and $P$. salicina exhibit a typical quadripartite structure with conserved genome arrangement, structure, and divergence and are similar to those of $P$. persica and $P$. pseudocerasus. The chloroplast genome size is $\sim 157 \mathrm{~kb}$ in Prunus species, including a pair of IRs separated by an LSC region and an SSC region (Table 1 and Fig. 2). The GC content of these Prunus chloroplast genomes is $~ 37 \%$ (Table 1); the GC content of the IR regions is $\sim 43 \%$ and those of LSC and SSC regions $\sim 35 \%$ and 30\%, respectively. These results lead us to infer that the LSC, SSC, and IR regions of five Prunus species are similar but that the GC contents are higher in IR regions due to the high GC contents of eight rRNA genes distributed in these regions.

The chloroplast genes of Prunus species contain 133 genes (110 unique genes), including 94 protein-coding, 31 tRNA, and eight rRNA genes (Table 1 and Table 2). There are 18 duplicated genes, including four rRNA genes and 13 other genes $(y c f 2, y c f 15, r p l 2$, rps19, trnI-CAT, rpl23, trnL$C A A, n d h B, r p s 7, r p s 12$, trnV-GAC, trnR-ACG, and trnN$G T T)$ repeats once where the ycf15 gene repeats twice in the IR region. Furthermore, 12 intron-containing genes were found (Table 3), including nine different genes ( $p s a A$, atpF, rpl22, ndhA, ndhB, rpoC1, trnS-AGA, rpl2, and ycf15) containing one intron and trnI-TAT in P. salicina and $P$. mume. The trnI-ATA gene in P. armeniaca also contains one intron. Two genes $(y c f 3$ and $c l p P)$ have two introns. These nine introns are located in the LSC region; two introns are in the SSC region and three introns in the IR region. The complete chloroplast genome with gene annotations has been submitted to NCBI under GenBank accession numbers MH700953 for P. mume, MH700954 for P. armeniaca, and MH700952 for P. salicina. 
Table 1 Summary statistics for the assembly of five Prunus species chloroplast genomes

\begin{tabular}{|c|c|c|c|c|c|}
\hline Genome features & P. armeniaca & P. mume & P. salicina & P. pseudocerasus & P. persica \\
\hline Genome size (bp) & 157,797 & 157,815 & 157,916 & 157,834 & 157,790 \\
\hline LSC size (bp) & 86,283 & 86,113 & 86,122 & 85,964 & 85,968 \\
\hline SSC size (bp) & 18,734 & 18,916 & 19,028 & 19,084 & 19,060 \\
\hline IR size (bp) & 26,390 & 26,393 & 26,383 & 26,393 & 26,381 \\
\hline Number of genes & $133(110)$ & $133(110)$ & $133(110)$ & $131(111)$ & $130(110)$ \\
\hline Protein genes [unique] & $94(80)$ & $94(80)$ & $94(80)$ & $86(78)$ & $85(77)$ \\
\hline tRNA genes [unique] & $31(26)$ & $31(26)$ & $31(26)$ & $37(29)$ & $37(29)$ \\
\hline rRNA genes [unique] & $8(4)$ & $8(4)$ & $8(4)$ & $8(4)$ & $8(4)$ \\
\hline Duplicated genes in IR & 18 & 18 & 18 & 16 & 14 \\
\hline GC content (\%) & 36.75 & 36.74 & 36.74 & 37 & 37 \\
\hline GC content in LSC (\%) & 34.54 & 34.58 & 34.58 & 35 & 35 \\
\hline GC content in SSC (\%) & 30.43 & 30.35 & 30.40 & 30 & 30 \\
\hline GC content in IR (\%) & 42.57 & 42.56 & 42.59 & 43 & 43 \\
\hline Total reads & $23,517,590$ & $44,218,598$ & $23,901,827$ & - & - \\
\hline Aligned paired-end reads & 662,524 & $1,107,094$ & 824,216 & - & - \\
\hline Assembled reads & 362,494 & 468,122 & 419,364 & - & - \\
\hline Average organelle coverage & 634 & 1059 & 788 & - & - \\
\hline Average insert size (bp) & 296 & 297 & 298 & - & - \\
\hline
\end{tabular}

Shrinkage and expansion of the IR region is an important aspect of the chloroplast genome, which is the main reason for the different sizes of these genomes. The IR regions of the five species of Prunus are shown in Fig. 2. The gene content and arrangement of the five species are the same in the IR region, which is extended in the rps19 and $y c f 1$ genes. The rps19 gene, located at the boundary of the LSC/IRa region of the five Prunus species, shows the same fragment size of $278 \mathrm{bp}$ in all species. In the LSC region, the fragment size ranges from 87 to $90 \mathrm{bp}$; in the SSC region, the fragment size ranges from 188 to $191 \mathrm{bp}$. The difference in the boundary region is one of the main reason for differences in chloroplast genome sizes. In addition, the IRa/SSC boundary is crossed by the $n d h F$ gene, with equal distributions in $P$. mume and $P$. armeniaca of $18 \mathrm{bp}$ in IRa, and $2219 \mathrm{bp}$ in SSC. The $n d h F$ gene in the $P$. salicina chloroplast genome spans the boundary of the IRa/SSC region, with $3 \mathrm{bp}$ more than in $P$. armeniaca and $P$. mume. Based on IRa/LSC and IRa/SSC boundaries, the relationship between $P$. mume and $P$. armeniaca is closer than that between $P$. mume and $P$. salicina. At the SSC/IRb border, $y c f 1$ is a critical gene that spans the IRb region and the SSC region in $P$. salicina and $P$. mume. The sizes of the fragments in the SSC regions of $P$. mume and P. salicina are $8 \mathrm{bp}$ and $4584 \mathrm{bp}$, respectively. The size of the $y c f 1$ gene fragment located in the
IRb region is $4494 \mathrm{bp}$ in $P$. mume and $1052 \mathrm{bp}$ in $P$. salicina. The $y c f 1$ gene located at the SSC region is only 87 bp from the SSC/IRb boundary in P. armeniaca. Furthermore, the trnN-GTT gene located in the IRb region is $1378 \mathrm{bp}$ from the critical point. At the IRb/LSC boundary, the rps 19 gene spans two regions in the three species with similar fragment sizes between the two regions. The fragment sizes of $P$. mume, $P$. salicina, and $P$. armeniaca are 192, 186, and $189 \mathrm{bp}$; those of the LSC region are 11, 2, and $2 \mathrm{bp}$, respectively. By comparing the IRb/SSC and $\mathrm{LSC} / \mathrm{IRb}$ regions of $r p s 19, n d h F$, and $y c f 1$, significant differences in fragment lengths of SSC and IRb regions were found among the three species.

\section{Repeat sequence and codon analysis}

REPuter software was used to identify a large number of repeat sequences in the chloroplast genome of Prunus species (Table 4). These repeats are distributed from 20 to $40 \mathrm{bp}$ in the gene spacer ( $p s b T$ to $p s b N$, trnT to TGT-trnFGAA, psbI to trnS-GCT, and rps19 to trnH-GTG), the coding region (rps12, $n d h K, \operatorname{trnS-TGA,~and~} n d h C)$, introns (ndhA, trnS-AGA, trnS-AGA, and $Y c f 3$ ) and other regions. In particular, the $y c f 3$ intron region and the rps19-trnH-GTG spacer region exhibit multiple nested sequence repeats. The chloroplast genomes of $P$. mume, P. armeniaca, and P. salicina have 10, 14, and 12 forward 


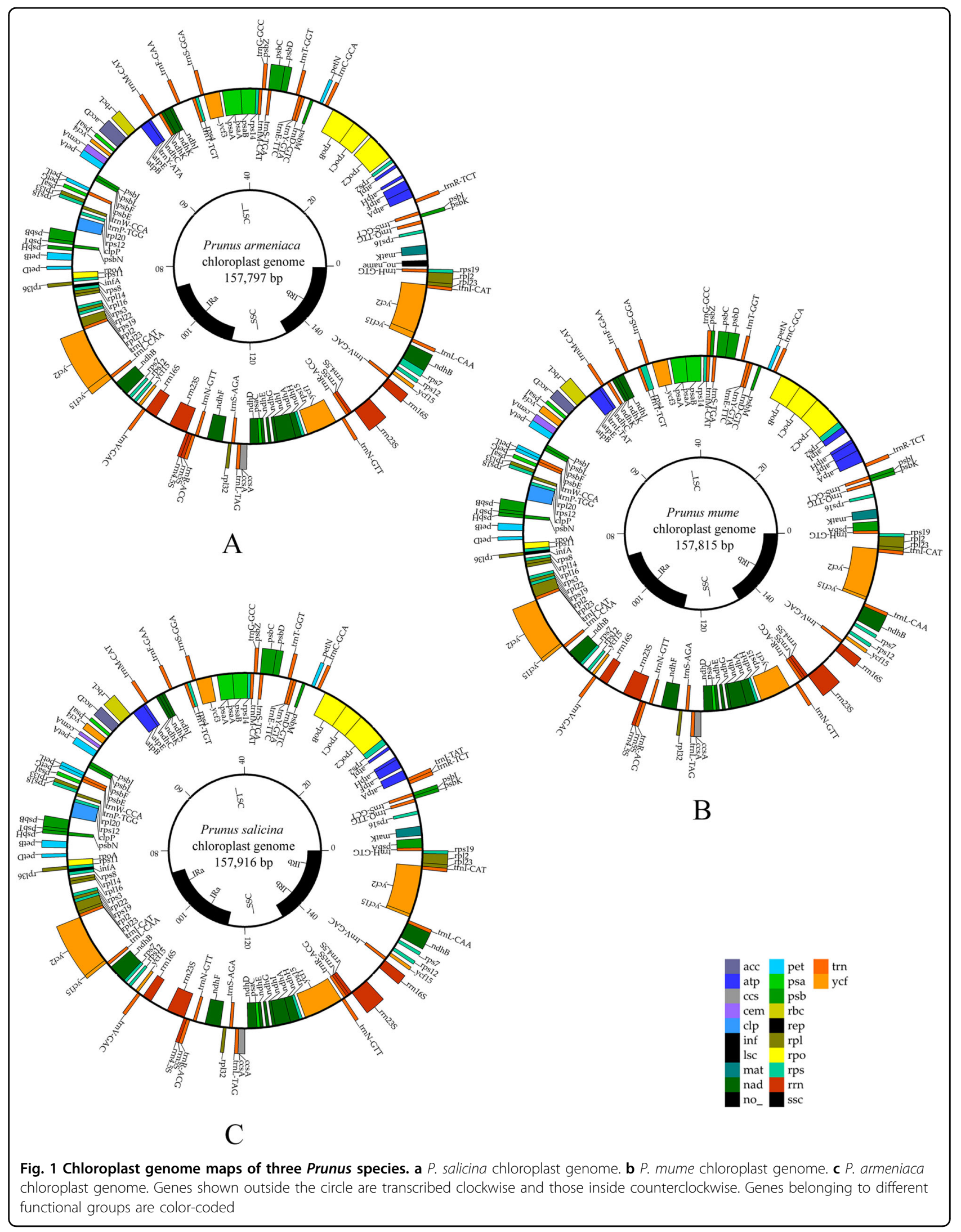




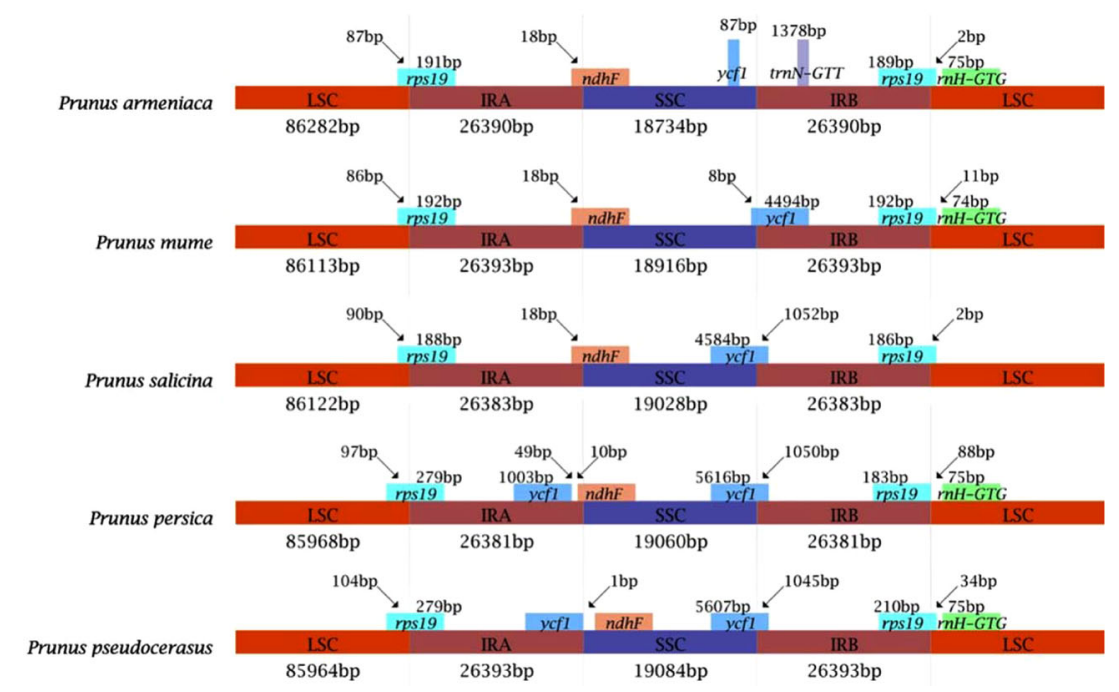

Fig. 2 Comparison of the borders of LSC, SSC, and IR regions of chloroplast genomes in five Prunus species

repeats, 13, 13, and 15 palindrome repeats, and 11, 12, and 11 reverse repeats, respectively, with no complementary repeats. The length distribution of the repeat sequence is mainly 20-24 bp and rarely 35-39 bp among $P$. mume, $P$. salicina, and $P$. armeniaca (Fig. 3). However, a significant difference among these five accessions, $P$. persica and $P$. pseudocerasus was found, whereby the difference in the number of repeats among the three species is greatest at 20-24 bp repeats, whereas $P$. persica and $P$. pseudocerasus have the highest number of repeats at 30-34 bp.

The software CodonW was used to calculate and analyze relative synonymous codon usage (RSCU) and bias in the chloroplast genomes. The protein-coding sequences of the $P$. mume, $P$. salicina, and $P$. armeniaca chloroplast genomes consist of 27,632, 27,113, and 28,043 codons, respectively. Among encoded amino acids, leucine is most frequent and tryptophan least frequent. The codon usage bias is related to the genetic information of the ancestral vector, DNA, and proteins involved in biological processes.

\section{Association analysis between different genes, hotspots, and simple sequence repeats (SSRs)}

A comparison of the chloroplast genomes of Prunus species suggests a difference in gene arrangement and content, with the trnl-TAT gene ranking 10th in the $P$. salicina chloroplast genome and 44th in P. mume; in contrast, this gene was not found in $P$. armeniaca. trn $Y$ $A T A$ is a unique gene in the $P$. armeniaca chloroplast genome, corresponding to the $y c f 1-\operatorname{atp} B$ differential region in the $52-53 \mathrm{k}$ region of the comparison map. Furthermore, trnH-GTG is at the last position in the $P$. armeniaca chloroplast gene, though it is at the first position in $P$. mume and $P$. salicina. A trnH-GTG-matK hotspot is present in the $0-3 \mathrm{k}$ region. These two differences can be the basis for molecular markers and species identification.

Using MISA software, we also found 59, 54, 49, 57, and 57 SSRs of at least $10 \mathrm{bp}$ in $P$. armeniaca, $P$. mume, $P$. salicina, $P$. persica, and $P$. pseudocerasus, respectively (Table 4, Fig. 4). Among these SSRs, most are located in the LSC/SSC region; the IRa and IRb regions have only one SSR. $P$. armeniaca has ten more SSRs than does $P$. salicina. Only single-, double-, and complex-nucleotide SSRs were detected in these Prunus species, though no three- or four-nucleotide SSRs were detected. Singlenucleotide repeats in $P$. mume, $P$. armeniaca, and $P$. salicina account for the total number of SSRs, at $90.74 \%$, $89.66 \%$, and $89.80 \%$, respectively. The high variation of SSRs in the chloroplast genome has excellent value for molecular marker studies and plant breeding.

We divided these regions into three grades based on the degree of difference (Fig. 5). The first grade has significant differences among the 26 Rosaceae chloroplast genomes and includes rps16-atpA, atpH-atpI, trnc-GCA-psbD, $y c f 3-a t p B$, and $r p L 32-n d h D$, which can be used as focus regions for the development of and molecular marker studies in Rosaceae fruit. The second grade is the significant difference in a portion of the 26 Rosaceae chloroplast genomes, including trnH-GTG-matK, PsbZ$P s b B, r b c L-a c c D, p s a I-c e m A, p s b J-p s b B, p s b T-r p s 3, n d h G-$ $n d h H$, and $r p s 15-y c f 1$, which can be considered as hotspots for the research and development of molecular markers of Rosaceae. The third grade, comprising the four regions trnL-CAT-ycf15, trnN-GTT, trnR-GTT-ndhF, and $n d h B$, displays a partial difference among Rosaceae. These 
Table 2 List of annotated genes in P. mume, P. armeniaca, and P. salicina chloroplast genomes

\begin{tabular}{|c|c|c|}
\hline Category & Group of gene & Name of gene \\
\hline \multirow[t]{6}{*}{ Photosynthetic } & Subunits of photosystem I & $p s a A(x 2), p s a B, p s a C, p s a l, p s a J$ \\
\hline & Submits of photosystem II & $p s b A, p s b B, p s b C, p s b D, p s b E, p s b F, p s b H, p s b l, p s b J, p s b K, p s b L, p s b M, p s b N, p s b T, p s b Z$ \\
\hline & Subunits of NADH dehydrogenase & $n d h A, n d h B(x 2), n d h C, n d h D, n d h E, n d h F, n d h G, n d h H, n d h 1, n d h J, n d h K(x 2)$ \\
\hline & Subunits of cytochrome b/f complex & petA, petB, petD, petG, petL, pet $N$ \\
\hline & Subunits of ATP synthase & $\operatorname{atp} A, \operatorname{atp} B, \operatorname{atp} E, \operatorname{atpF}, \operatorname{atpH}, \operatorname{atpl}$ \\
\hline & Large subunit of rubisco & $r b c L$ \\
\hline \multirow[t]{5}{*}{ Self-replication } & Proteins of large ribosomal subunit & $r p / 2(x 2), r p / 14, r p / 16, r p / 20, r p / 22, r p / 23(x 2), r p / 32, r p / 33, r p / 36$ \\
\hline & Proteins of small ribosomal subunit & $r p s 2, r p s 3, r p s 4, r p s 7(x 2), r p s 8, r p s 11, r p s 12(x 3), r p s 14, r p s 15, r p s 16, r p s 18, r p s 19(\times 2)$ \\
\hline & Subunits of RNA polymerase & rpoA, rpoB, rpoC1, rpoC2 \\
\hline & Ribosomal RNAs & $r r n 23 S(x 2), r r n 16 S(x 2), r r n 5 S(x 2), r r n 4.5 S(x 2)$ \\
\hline & Transfer RNAs & $\begin{array}{l}\operatorname{trn} R-T C T, \operatorname{trn} Y-A T A^{*}, \operatorname{trn} C-G C A, \operatorname{trn} T-G G T, \operatorname{trn} I-C A T(x 2), \operatorname{trn} S-G G A, \operatorname{trn} F-G A A, \operatorname{trn} M-C A T, \\
\operatorname{trn} G-G C C, \operatorname{trn} R-A C G(x 2), \operatorname{trn} L-T A G, \operatorname{trn} H-G T G, \operatorname{trn} Y-G T A, \operatorname{trn} P-T G G, \operatorname{trn} V-G A C(\times 2), \operatorname{trn} S-G C T, \\
\operatorname{trn} S-A G A, \operatorname{trn} Q-T T G, \operatorname{trn} D-G T C, \operatorname{trn} L-C A A(x 2), \operatorname{trn} W-C C A, \operatorname{trn} T-T G T, \operatorname{trn} F-C A T, \operatorname{trn} S-T G A, \\
\operatorname{trn} N-G T(\times 2), \operatorname{trn} E-T T C\end{array}$ \\
\hline \multirow[t]{6}{*}{ Biosynthesis } & Maturase & matK \\
\hline & Protease & $c l p P$ \\
\hline & Envelope membrane protein & cemA \\
\hline & Acetyl-CoA carboxylase & $\operatorname{acc} D$ \\
\hline & c-type cytochrome synthesis gene & $\operatorname{ccs} a(x 2)$ \\
\hline & Translation initiation factor & $\inf A$ \\
\hline Unknown function & $\begin{array}{l}\text { Conserved hypothetical chloroplast } \\
\text { Reading Frames }\end{array}$ & $y c f 1, y c f 2(x 2), y c f 3, y c f 4, Y c f 15(x 4)$ \\
\hline
\end{tabular}

Asterisk denotes the trnY-ATA gene is trnY-ATA in P. armeniaca but is trnl-TAT in P. mume and P. salicina

Table 3 Information on 12 intron-containing genes in the chloroplast genome of Prunus species

\begin{tabular}{|c|c|c|c|c|c|c|}
\hline Gene & Location & Exon I (bp) & Intron I (bp) & Exon II (bp) & Intron II (bp) & Exon III (bp) \\
\hline trnl-TAT & LSC & 38 & 83 & 42 & & \\
\hline psaA & LSC & 1787 & 31 & 323 & & \\
\hline ycf3 & LSC & 126 & 713 & 229 & 764 & 149 \\
\hline $\operatorname{atpF}$ & LSC & 147 & 683 & 466 & & \\
\hline rp/2 & $\mathbb{R}$ & 384 & 648 & 469 & & \\
\hline ycf15 & $\mathbb{R}$ & 200 & 295 & 110 & & \\
\hline$c l p P$ & LSC & 73 & 805 & 296 & 648 & 222 \\
\hline rp/22 & LSC & 380 & 64 & 123 & & \\
\hline$n d h A$ & SSC & 555 & 1147 & 535 & & \\
\hline$n d h B$ & $\mathbb{R}$ & 869 & 588 & 752 & & \\
\hline rpoCl & LSC & 455 & 755 & 1613 & & \\
\hline $\operatorname{trnS}-A G A$ & SSC & 48 & 77 & 34 & & \\
\hline
\end{tabular}


Table 4 Summary of repeat sequences and SSRs in five Prunus accessions

\begin{tabular}{|c|c|c|c|c|c|}
\hline Species & P. armeniaca & P. mume & P. salicina & P. persica & P. pseudocerasus \\
\hline Total number & 39 & 34 & 38 & 48 & 49 \\
\hline Forward & 14 & 10 & 12 & 18 & 19 \\
\hline Palindromic & 13 & 13 & 15 & 20 & 20 \\
\hline Reverse & 12 & 11 & 11 & 10 & 10 \\
\hline SSR loci (N) & 59 & 54 & 49 & 57 & 57 \\
\hline $\mathrm{P} 1^{\mathrm{a}}$ locia (N) & 52 & 49 & 44 & 51 & 48 \\
\hline $\mathrm{P} 2^{\mathrm{b}}$ loci $(\mathrm{N})$ & 4 & 4 & 1 & 3 & 2 \\
\hline$P C^{c}$ loci $(\mathrm{N})$ & 3 & 1 & 4 & 3 & 7 \\
\hline LSC & 51 & 47 & 41 & 49 & 48 \\
\hline SSC & 6 & 5 & 6 & 6 & 7 \\
\hline IRa & 1 & 1 & 1 & 1 & 1 \\
\hline $\mathrm{IRb}$ & 1 & 1 & 1 & 1 & 1 \\
\hline
\end{tabular}

${ }^{\mathrm{a}}$ single-nucleotide SSRs

bouble-nucleotide SSRs

complex-nucleotide SSRs

17 regions are generally rich in SSRs, for example, rps16atpA in first-grade hotspots contain six $\left[(\mathrm{A})_{10},(\mathrm{~A})_{12}\right.$, $(\mathrm{A})_{10},(\mathrm{~T})_{10},(\mathrm{~T})_{16}$, and $\left.(\mathrm{T})_{10}\right]$, six $\left[(\mathrm{A})_{17},(\mathrm{~A})_{12},(\mathrm{~A})_{12},(\mathrm{~A})_{10}\right.$, $(\mathrm{T})_{11}$, and $\left.(\mathrm{T})_{11}\right]$ and six $\left[(\mathrm{A})_{15},(\mathrm{~A})_{17},(\mathrm{~A})_{10},(\mathrm{~T})_{10},(\mathrm{~T})_{10}\right.$, and $\left.(\mathrm{T})_{10}\right]$ SSRs in P. mume, P. salicina, and P. armeniaca, respectively. The atpH-atpI hotspots have three $\left[(\mathrm{A})_{11}\right.$, $(\mathrm{T})_{12}$, and $\left.(\mathrm{T})_{11}\right]$, two [(T) $)_{11}$ and $\left.(\mathrm{T})_{11}\right]$, and two $\left[(\mathrm{T})_{10}\right.$ and $(\mathrm{T})_{11}$ ] SSRs in $P$. mume, $P$. salicina, and $P$. armeniaca, respectively. The trnc-GCA-psbD hotspots have two $\left[(\mathrm{T})_{10}\right.$ and $\left.(\mathrm{T})_{10}\right]$, one $\left[(\mathrm{A})_{12}\right]$, and two [(A $)_{12}$ and $\left.(\mathrm{T})_{10}\right]$ SSRs in $P$. mume, $P$. salicina, and $P$. armeniaca, respectively. The $y c f 3$-atpB hotspots have four $\left[(\mathrm{AT})_{7}\right.$ aaa $(\mathrm{AT})_{6},(\mathrm{~T})_{10},(\mathrm{~A})_{15}$, and $\left.(\mathrm{T})_{10}\right]$, five $\left[(\mathrm{T})_{11},(\mathrm{~T})_{10},(\mathrm{~A})_{12},(\mathrm{TA})_{7}\right.$, and $\left.(\mathrm{T})_{10}\right]$ and five $\left[(\mathrm{T})_{11},(\mathrm{~A})_{12},(\mathrm{TA})_{6},(\mathrm{~T})_{10}\right.$, and $\left.(\mathrm{ATA})_{5} \operatorname{tact}(\mathrm{ATA})_{5}\right]$ SSRs in $P$. mume, $P$. salicina, and $P$. armeniaca, respectively. The $r p L 32-n d h D$ hotspots have two [(A)10taaaatatttttcttaattaattatttctgattcaccggttcttatttgttttctgtt-

gaaaggggtcagttaat(A)10 and $\left.(\mathrm{A})_{15}\right]$, one $\left[(\mathrm{A})_{14}\right]$, and one $\left[(\mathrm{A})_{14}\right]$ SSRs in the three species. The other two grades are also similar and contain abundant SSR molecular markers, and their distribution is positively related.

In conclusion, the difference in the sequence of the IR region is smaller than that in the LSC and SSC regions. The coding region of the gene is more conserved than is the noncoding region, and the rRNA region is also conserved. Furthermore, the intron region shows the highest mutation rate, followed by the LSC region, the chloroplast genome, the SSC region, and the proteincoding region, with a slight change in the IR region. The distribution of SSRs is positively related to differential hotspots.

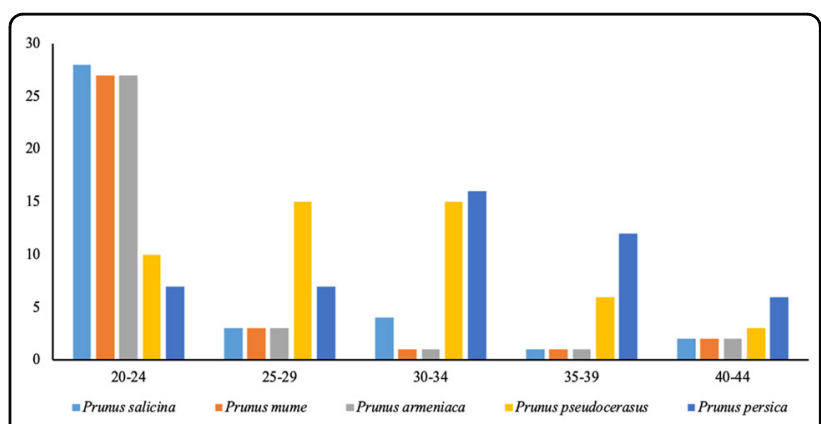

Fig. 3 Length distribution of repeat sequences in Prunus species

\section{Chloroplast phylogenetic analysis}

Phylogenetic relationships of the Rosaceae family and taxonomic statuses were systematically classified through maximal parsimony analysis of three complete chloroplast sequences. In this study, we combined 23 published complete chloroplast genomes and the chloroplast genomes of $P$. mume, $P$. salicina, and $P$. armeniaca. Thus, a total of 26 species were used to reconstruct a phylogenetic tree using MEGA7 software. We utilized different data, including the complete chloroplast genome and CDS, LSC, IR, and intron regions to construct the phylogenetic tree (Fig. 6). The phylogenetic trees constructed with complete chloroplast genome, CDS, and LSC data have the same topology, whereas the trees constructed from IR and intron datasets have low reliability. The phylogenetic trees based on the complete chloroplast genome, CDS, LSC, IR, and intron data have high bootstrap values (this value is generally considered to be a more stable branch 

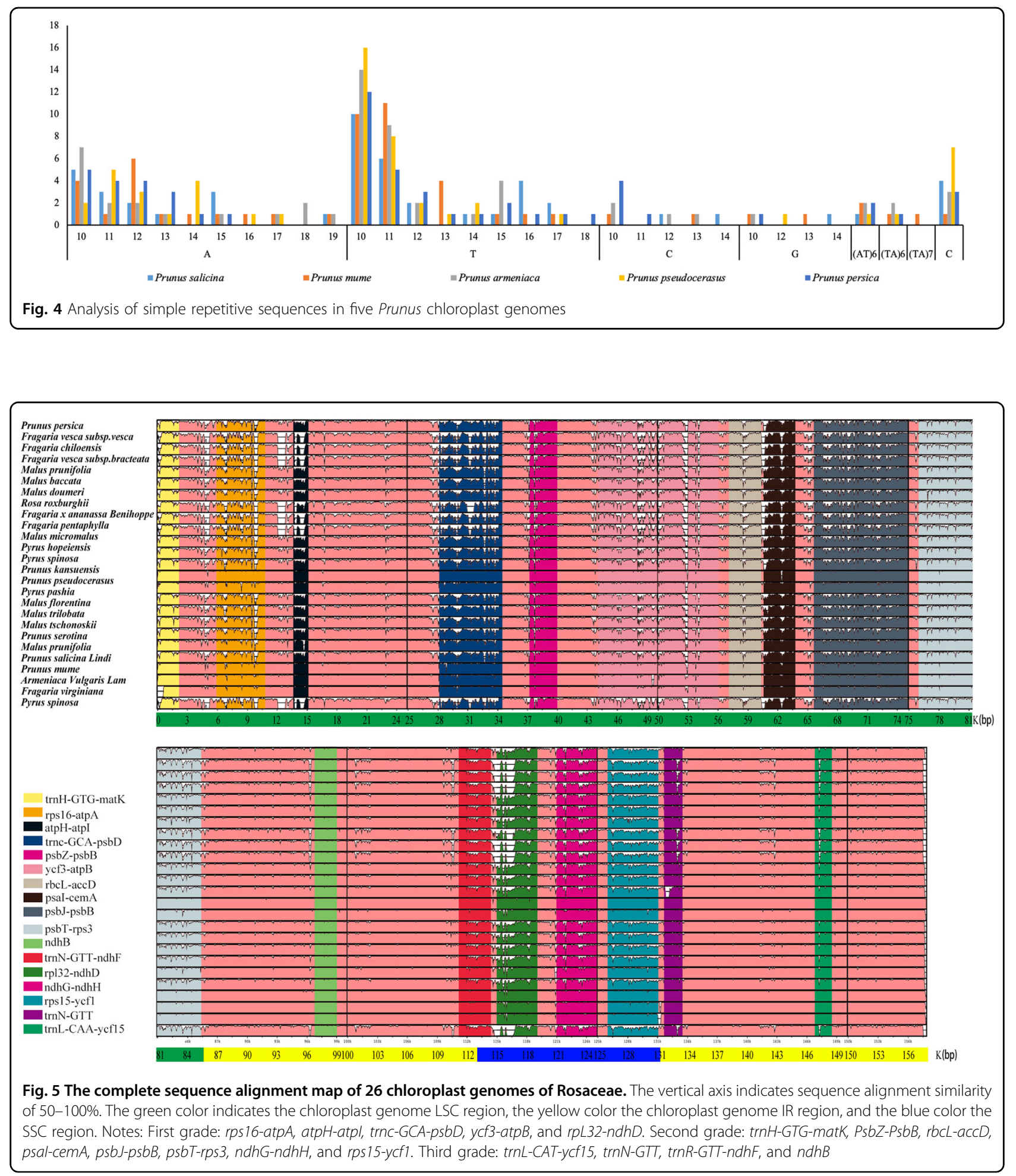

than 75). The higher is the branch's credibility, the more consistent is the guiding value of the evolutionary analysis for the relationship. Furthermore, the phylogenetic trees suggest that $P$. mume, $P$. salicina, and $P$. armeniaca form a single group. Our results showed that the genera Malus,
Prunus, Fragaria, and Rosa form one branch. Malus and P. salicina are divided into a taxonomic division, whereas Fragaria and Rosa belong to a branch that requires further verification. In addition, the phylogenetic trees constructed based on chloroplast genome, CDS, and LSC data 

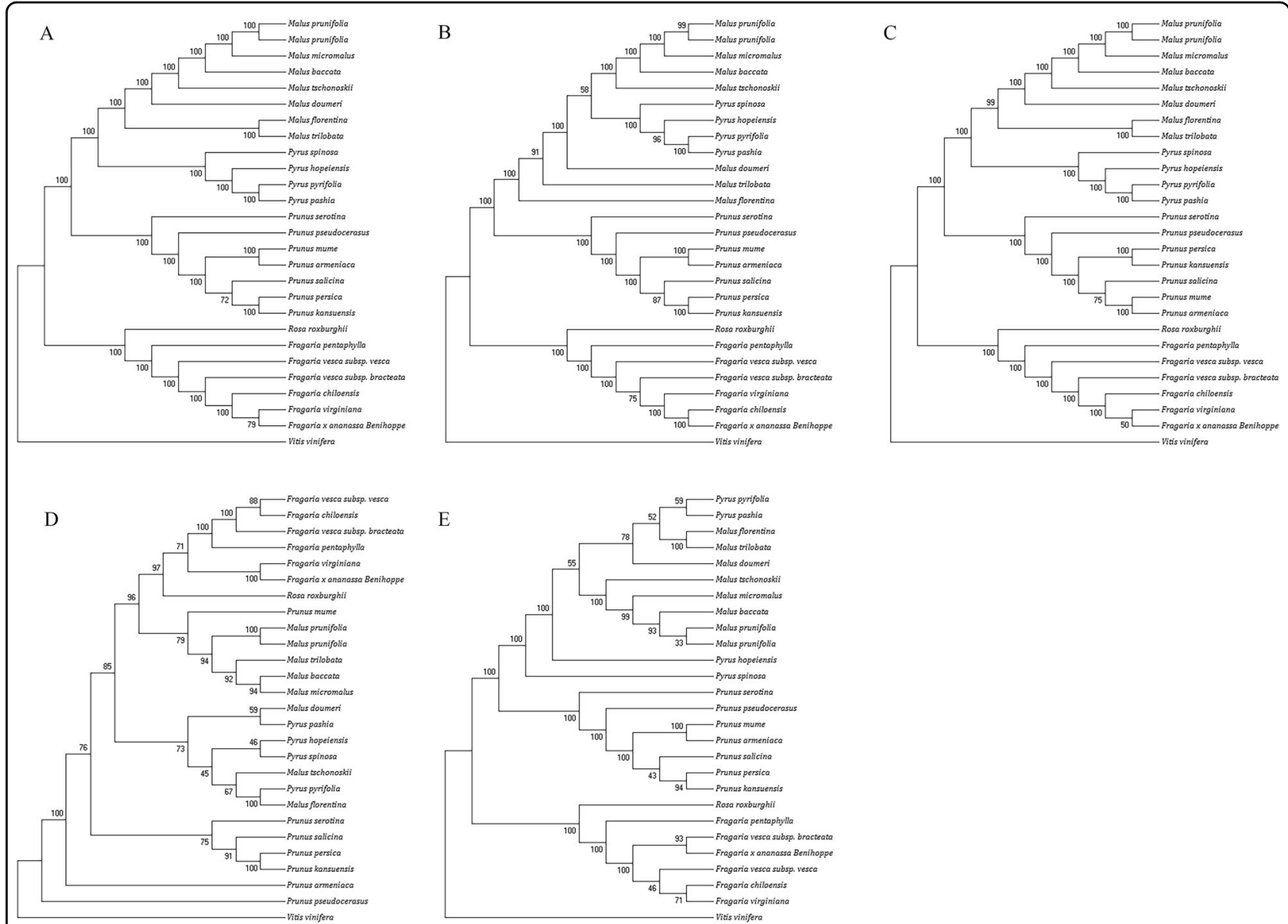

Fig. 6 Phylogenetic trees of the Prunus species based on the chloroplast genome by MP. a Phylogenetic tree constructed using the complete chloroplast genome data. b Phylogenetic tree constructed using coding region data. c Phylogenetic tree constructed using LSC data. d Phylogenetic tree constructed using intron data. e Phylogenetic tree constructed using IR data

showed that $P$. mume, $P$. salicina, and P. armeniaca are closer to each other than to other Rosaceae species. The tree branch length of $P$. salicina is long, but those of $P$. mume and $P$. armeniaca are similar; the evolutionary differences between $P$. mume, $P$. salicina, and $P$. armeniaca are pronounced. $P$. mume is closer to $P$. armeniaca than to $P$. salicina.

\section{Discussion}

The chloroplast genome of most angiosperm species contains 74 protein-coding genes, though some have 79 protein-coding genes ${ }^{16}$. Previous studies on Rosaceae fruit trees have revealed that chloroplast gene numbers range from 110 to $130^{17}$. In this study, sequence analysis revealed 133 genes (110 unique genes), including 94 protein-coding, 31 tRNA, and 8 rRNA genes. The chloroplast genome among Prunus species is similar in intron and GC contents, but the GC contents in LSC and SSC regions are significantly lower than that in the IR region. The main reason for this is that all eight rRNA genes with high GC contents are distributed in the IR region. In general, the IR region is the most conserved region of the chloroplast genome ${ }^{18}$. Expansion and contraction in IR, LSC, and SSC regions are common during evolution and are the primary causes of differences in chloroplast genome lengths. A comparative map of the border regions of the chloroplast genome was obtained based on analysis of boundary genes between IR, LSC, and SSC regions. The difference in boundary region size is one of the main reasons for alterations among chloroplast fragments ${ }^{19,20}$.

The chloroplast genomes of the five species of Prunus are $\sim 157 \mathrm{~kb}$. $P$. salicina has the largest chloroplast genome at $157,916 \mathrm{bp}$. In general, there are three main reasons for a change in the size of the chloroplast genome ${ }^{16}$. The first is shrinkage, expansion, or loss of the IR region. Changes in the length of different chloroplast genomes are generally due to changes in the IR region and the boundary between the LSC and SSC regions. Goulding et al. $^{21}$ proposed a hypothesis for the evolution of the chloroplast IR region: there is a small amplification of the 
boundary gene in the IR zone and recombinant repair of the LSC boundary. Small amplification of the boundary genes in the IR zone is considered to be an important factor in maintaining the stability of the IR zone $\mathrm{e}^{22}$. The second is loss or increases in genes in the SSC region. The third reason is a decrease in the length of introns or the gene spacer region. For example, the loss of introns in the $y c f 1$ gene might be the main reason for the smaller chloroplast genome of $P$. salicina. Intron loss was also reported for Welwitschia mirabilis, Hordeum vulgare, and Manihot esculenta ${ }^{23,24}$.

In our study, changes in the IR and boundary between the IR and LSC or SSC of the chloroplast genome of three species were found to be small. The rps19 gene spans LSC-IR and SSC-IR boundaries, similar to the crossing of LSC-IR and SSC-IR boundaries in the chloroplast genomes of Ilex pubescens, Helwingia himalaica, and Panax ginseng $^{25}$. In some species, the rps 19 gene is located at the border of the LSC/IRa region in the chloroplast genome, as in the genus Fragaria; ${ }^{19}$ the boundary in Asteraceae, such as Millettia pinnata ${ }^{26}$ and Lupinus luteus, is close but does not extend into the IR. In the case of other species, such as Phaseolus vulgaris ${ }^{27}$ and Vigna radiata ${ }^{28}$, the entire gene is present in the IR. The genes ycf1 and ndhF are closest to the SSC-IR border, similar to the case of the rps19 gene. There are reports of species with genes located on the border, across the border or in the IR region. Comparison between IR regions and the chloroplast genomes of $P$. armeniaca, $P$. mume, and P. salicina showed similarity, which was the same as for the boundary gene of LSC/IRa, IRa/SSC, and IRb/LSC regions and the size of genes and fragments in two adjacent regions. The results revealed high similarity among the three species. We also found that the situation at the IRa/ LSC boundary was almost the same in P. armeniaca and $P$. mume. Therefore, we speculate that the genetic relationship between $P$. armeniaca and $P$. mume is closer than that between $P$. mume and $P$. salicina, and our subsequent phylogenetic analysis validates our inference. We also found that the boundary of the SSC/IRb region displays the greatest difference among $P$. mume, $P$. salicina, and $P$. armeniaca and that is the most variable region.

In a previous report, 17 mutations were found in the cpDNA of 18 Prunus accessions via RFLP analysis. Seven mutations, including one length mutation, clustered densely within a region of $\sim 9.1 \mathrm{~kb}$, which includes $p s b A$ and $\operatorname{atp} A$, in the left border of a large single-copy region of Prunus cpDNAs. All of these length mutations occurred within the $9.1 \mathrm{~kb}$ region between $p s b A$ and atpA. This region might be an intramolecular recombinational hotspot in Prunus species ${ }^{29}$.

Differences in the order and content of chloroplast genomes have already been reported for Aquifoliales ${ }^{30}$,
Asterales $^{31}$, Bruniales, Apiales, Paracryphiales, and Dipsacales ${ }^{30}$. Additionally, the $\operatorname{trn} Y-A T A$ gene is a unique gene in $P$. armeniaca, whereas trnI-TAT has a different order in P. mume and P. salicina. trnI-TAT of P. salicina is located at the 10th position of the chloroplast genome, but this gene is at the 44th position in $P$. armeniaca. The trnH-GTG gene is situated last in the $P$. armeniaca chloroplast genome, but it is first in $P$. mume and $P$. salicina. Previous reports have been based on differences in $A c c d, c l c p$, and other protein-coding genes, but differences in tRNA genes were discovered first.

The variation in SSR copy numbers in chloroplasts represents an important molecular marker, i.e., cpSSRs, which are widely used in plant population genetics, polymorphism investigations, and evolutionary research. Zhang et al. ${ }^{32}$ used $10 \mathrm{cpSSRs}$ and 16 nuclear SSRs to explain the morphology and differentiation of 42 species of the subgenus Prunophora. In the chloroplast genomes of $P$. mume, $P$. salicina, and P. armeniaca, the number of SSRs was found to be significantly higher than that in other angiosperms, and the content of $\mathrm{A} / \mathrm{T}$ repeats is far greater than that of $\mathrm{G} / \mathrm{C}$ repeats, similar to the results of Melotto-Passarin and other studies ${ }^{33,34}$. In addition, SSR loci in these three species differ from those of strawberry, also belonging to Rosaceae, with three and four duplications found, which has also been found among other families such as Illex ${ }^{30}$ and Chinese Juglans ${ }^{35}$, and mononucleotides, dinucleotides, trinucleotides, tetranucleotides, pentanucleotides, and complex nucleotides have been detected in their chloroplast genomes. The single-nucleotide repeat in cpSSRs that we found can be used to detect polymorphisms at the population level and to compare long-range phylogenetic relationships of different species. Guisinger and Weng ${ }^{36,37}$ found that repetitive sequences might play an important role in chloroplast genome arrangement and sequence variation. In this study, we found a large number of repetitive sequences in the chloroplast genomes of $P$. mume, $P$. salicina, and $P$. armeniaca, especially in the intergenic region, which is consistent with the results of studies on the chloroplast genomes of Quercus ${ }^{38}$ and Holly ${ }^{39,40}$. These repetitive sequences can be used as important resources for studying differences in chloroplast genes.

Comparison of whole-genome sequences indicated that the different hotspots correlated positively with the distribution of SSRs and that specific genes are also present in hotspots. The different hotspots of plastids have been used to design molecular markers for phylogenetic relationships, such as $r b c L$, matK, and $a t p B$, which have been widely used in general phylogenetic studies ${ }^{30}$. The diversity of wild cherry, P. salicina, was analyzed using chloroplast markers, revealing a certain evolutionary relationship. Indeed, different regions of chloroplast are important for species-level identification of Rosaceae ${ }^{12,41}$. 
There are many species with similar traits in the family Rosaceae. By comparing chloroplast sequences, we can clearly observe differences in genomes between species at the molecular level and divide species based on chloroplast sequences. The difference in the IR region between the genera Malus, Fragaria, and Prunus is less than that in LSC and SSC regions. Moreover, coding regions are more conserved than are noncoding regions, and rRNA sequences are also conserved. The intron region showed the highest mutation rate, followed by the LSC region, the complete chloroplast genome, the SSC region, and the protein-coding region, with the IR region having the smallest rate. Sequence variations in $P$. mume and $P$. armeniaca are smaller than those in $P$. salicina, similar to the results of the phylogenetic analysis. These hotspots are important molecular marker resources for phylogenetic analysis and identification of Rosaceae plants ${ }^{42}$.

Phylogenetic relationships in Rosaceae have long been problematic because of frequent hybridization, apomixis, presumed rapid radiation, and historical diversification. Plastid phylogenomics offers novel and deep insight into phylogenetic relationships and diversification history among Rosaceae. The development of chloroplast phylogeny and time estimation provides new evidence for future comparative evolutionary studies ${ }^{43}$. Phylogenetic analysis using the chloroplast genome sequence is applied to evaluate evolutionary relationships of species. Our phylogenetic tree was based on complete chloroplast genome, CDS, LSC, IR, and intron data, and the results are consistent with the traditional classification system, indicating that the classification of Rosaceae is generally reasonable. The results of our phylogenetic analysis partially agree with the traditional classification system of Chinese flora, e.g., the genera Rosa and Fragaria. This suggests that Rosa and Fragaria are closely related at the molecular level. The fruit, appearance, shape, and other characteristics of $P$. mume are very similar to those of $P$. salicina, though the taste and fragrance are very similar to those of $P$. armeniaca. However, the plants are more resistant to disease. Our phylogenetic tree suggests that $P$. armeniaca is closer to $P$. mume than to $P$. salicina, supporting the grouping of $P$. mume into $P$. armeniaca. With the emergence of more complete chloroplast genome sequences, the chloroplast genome is also expected to help resolve deeper branches of phylogeny. Although there are differences in the phylogenetic tree structure and molecular phylogeny of the Rosaceae family and relationship among various genera, these chloroplast genome sequences will provide genetic information for understanding the evolution of the plastid genome ${ }^{44}$.

\section{Conclusion}

The chloroplast genome size, GC content, and gene number, and order among three Prunus species (P. mume,
P. salicina, and $P$. armeniaca) are highly similar to each other. However, there are differences in SSC/IR and LSC/ IR boundaries and in the genes rps19 and $y c f 1$, with different expansion lengths in different species. When compared with other genetically related Rosaceae fruit trees, a total of 17 hot spots with significant differences were identified and can be used for the development of phylogenetic markers. The phylogenetic trees were constructed based on chloroplast genome, CDS, LSC, IR, and intron datasets, supporting the close relationship between $P$. mume and $P$. armeniaca. The phylogeny of Rosaceae was comprehensively analyzed. Our results provide a basis for identifying and overcoming phylogenetic problems at the species level.

\section{Materials and methods \\ Plant material}

We used $P$. mume, $P$. armeniaca, and $P$. salicina for genome sequencing. Young, healthy fresh leaves of $P$. mume and $P$. salicina were collected from the National Field Genebank for P. mume, Nanjing, Jiangsu Province, China, and fresh leaves of $P$. armeniaca were obtained from Jiangsu Institute of Agricultural Sciences, Nanjing, Jiangsu Province, China. All samples were immediately frozen in liquid nitrogen and stored at $-80^{\circ} \mathrm{C}$.

\section{Chloroplast genome sequencing and assembly}

Total genomic DNA was extracted from $100 \mathrm{mg}$ of fresh leaves using a modified CTAB (cetrimonium bromide) method. The DNA concentration $\left(>50 \mathrm{ng} \mathrm{LL}^{-1}\right)$ was measured using a NanoDrop spectrophotometer, and fragmentation was achieved using sonication. The fragmented DNA was purified and end-repaired, and sizes were determined by gel electrophoresis. The PCR products were used to produce short-insert (300 bp) libraries using Illumina Nextera XT and, subsequently, a control library quality for sequencing. We sequenced (based on sequencing by synthesis, SBS, technology) the complete chloroplast genome of the three Prunus species using the HiSeq $^{\text {nx }}$ X10 platform (Illumina, USA) (Genepioneer Biotechnologies Co. Ltd, Nanjing, Jiangsu, China). Raw reads were filtered using the base quality control software NGSQCToolkit v2.3.3 to obtain high-quality reads. We assembled the chloroplast genomes with NOVOPlasty using clean data and annotated them with CpGAVAS ${ }^{36}$. The technology used in this study comprised a combination of de novo sequencing with the Prunus persica chloroplast genome as a reference (NCBI accession number NC_014697.1). Finally, Sanger sequencing was used to verify LSC/IR and SSC/IR junctions.

\section{Genome annotation and sequence alignment}

The chloroplast genome sequences were assembled and annotated using the software Dual Organellar Genome 
Annotator ${ }^{44}$, coupled with manually edited start and stop codons. The three Prunus species chloroplast genome maps were drawn in Organellar Genome DRAW ${ }^{45}$, including the two previously sequenced chloroplast genome sequences from $P$. persica and P. pseudocerasus, and our three sequences were aligned by MAFFTv7.0.0 to identify the locations of introns and exons, putative start codons, and stop codons; sequences were then manually edited. Base content was analyzed with Bio-Edit software, and the genome annotation included genes, proteincoding genes, tRNA genes, introns, exons, and intergenic spacers; RSCU was analyzed with MEGA 7 software. We used REPuter (http://bibiserv.techfak.uni-bielefeld.de/ reputer/) to find and analyze the sizes and locations of forward, reverse, palindromic, and complementary repeats with a minimal length of $20 \mathrm{bp}$, an identity of $90 \%$ and a Hamming distance of $3^{46}$. SSRs were identified using MISA (http://pgrc.ipk-gatersleben.de/misa/), with thresholds for mononucleotide SSRs of ten repeats and dinucleotide and hexanucleotide SSRs of five repeats. We used CodonW Software to analyze codon usage bias.

Sequence alignment analysis was performed using the online comparison tool mVISTA. We selected 23 Rosaceae plants with similar genetic relationships for blast searches in NCBI.

\section{Phylogenetic analysis}

The chloroplast genomes of 26 Rosaceae species with strong genetic relationships were selected for phylogenetic analysis, and the grape chloroplast genome (NC_007957.1) was selected as the outgroup. The chloroplast genomic sequences of the 23 Rosaceae downloaded from NCBI were manually annotated. We selected IR, LSC, SSC, CDS, and complete chloroplast genome sequences for phylogenetic analysis. Before constructing the phylogenetic tree, we performed multiple sequence alignment using MAFFT software ${ }^{47}$ to obtain aligned chloroplast genomes for phylogenetic analysis. We used complete chloroplast genome sequence, LSC, SSC, IR, and CDS data and maximum parsimony to construct the phylogenetic tree. An MPL analysis was performed using MEGA 7, and a bootstrap test was performed with 1000 repetitions to calculate the maximum parsimony bootstrap value with tree bisection-reconnection branch swapping. Twenty-six species were compared and phylogenetic evaluated.

\footnotetext{
Acknowledgements

We gratefully acknowledge financial support for this research from the National Natural Science Foundation of China (31772282), the Project for National Crop Resources Conservation and Sharing Platform (NICGR2018-98), the Six Talent Peaks Project in Jiangsu Province (NY068), China Postdoctoral Science Foundation (2018M640497), and Jiangsu Postdoctoral Science Research Foundation (2018K216C).
}

\section{Author contributions}

This study was designed by Z.G. Collection and identification of field material were performed by S.X., Z.S., and Z.N. Sample preparation and analysis were performed by S.X., W.L., D.Y., and X.H. Data analysis was conducted by S.X., T.S., and X.N. Authors T.S. and S.X. wrote the paper. S.I. modified the language. All the authors read and approved the final manuscript.

\section{Conflict of interest}

The authors declare that they have no conflict of interest.

Received: 18 January 2019 Revised: 21 May 2019 Accepted: 31 May 2019 Published online: 21 July 2019

\section{References}

1. Carbonell-Caballero, J. et al. A phylogenetic analysis of 34 chloroplast genomes elucidates the relationships between wild and domestic species within the genus. Mol. Biol. Evol. 32, 2015-2035 (2015).

2. Yang, J. B., Yang, S. X., Li, H. T., Yang, J. \& Li, D. Z. Comparative chloroplast genomes of camellia species. PLoS One 8, e73053 (2013).

3. Wu, W. et al. PCR-RFLP analysis of cpDNA and mtDNA in the genus Houttuynia in some areas of China. Hereditas 142, 24-32 (2005).

4. Dyall, S. D., Brown, M. T. \& Johnson, P. J. Ancient invasions: from endosymbionts to organelles. Science 304, 253-257 (2004).

5. Shinozaki, K. et al. The complete nucleotide sequence of the tobacco chloroplast genome: its gene organization and expression. EMBO J. 5, 2043-2049 (1986).

6. Palmer, J. D. Comparative organization of chloroplast genomes. Ann. Rev. Genet 19, 325-354 (1985).

7. $\mathrm{Ma}$, J. et al. The complete chloroplast genome sequence of Mahonia bealei (Berberidaceae) reveals a significant expansion of the inverted repeat and phylogenetic relationship with other angiosperms. Gene 528, 120-131 (2013).

8. Zhang, Y. et al. The complete chloroplast genome sequence of Taxus chinensis var. mairei (Taxaceae): loss of an inverted repeat region and comparative analysis with related species. Gene 540, 201-209 (2014).

9. Hallick, R. B. et al. Complete sequence of Euglena gracilis chloroplast DNA. Nucleic Acids Res. 21, 3537-3544 (1993).

10. Reith, M. \& Munholland, J. Complete nucleotide sequence of the Porphyra purpurea chloroplast genome. Plant Mol. Biol. Rep. 13, 333-335 (1995).

11. Wang, $\mathbf{X}$. et al. Organellar genome assembly methods and comparative analysis of horticultural plants. Hortic. Res. 5, 3 (2018).

12. Zhang, S. D. et al. Diversification of Rosaceae since the Late Cretaceous based on plastid phylogenomics. New Phytol. 214, 1355-1367 (2017).

13. Velasco, R. et al. The genome of the domesticated apple (Malus $\times$ domestica Borkh). Nat. Genet. 42, 833-839 (2010).

14. Hirakawa, $\mathrm{H}$. et al. Dissection of the octoploid strawberry genome by deep sequencing of the genomes of Fragaria species. DNA Res. 21, 169-181 (2014).

15. VanBuren, R. et al. The genome of black raspberry (Rubus occidentalis). Plant $J$. 87, 535-547 (2016).

16. Bock, R. \& Knoop, V. Genomics of chloroplasts and mitochondria, Vol. 35 (Springer Science \& Business Media, Netherlands: Springer, 2012).

17. Daniell, H., Lin, C. S., Yu, M. \& Chang, W. J. Chloroplast genomes: diversity, evolution, and applications in genetic engineering. Genome Biol. 17, 134 (2016).

18. Li, R., Ma, P. F., Wen, J. \& Yi, T. S. Complete sequencing of five araliaceae chloroplast genomes and the phylogenetic implications. PLoS One 8, e78568 (2013).

19. Cheng, $\mathrm{H}$. et al. The complete chloroplast genome sequence of strawberry (Fragaria $\times$ ananassa Duch.) and comparison with related species of Rosaceae. Peer J. 5, e3919 (2017)

20. Ni, L., Zhao, Z., Dorje, G. \& Ma, M. The complete chloroplast genome of YeXing-Ba (Scrophularia dentata; Scrophulariaceae), an alpine Tibetan herb. PLoS One 11, e0158488 (2016).

21. Goulding, S. E., Olmstead, R. G., Morden, C. W. \& Wolfe, K. H. Ebb and flow of the chloroplast inverted repeat. Mol. Gen. Genet. 252, 195-206 (1996).

22. Ravi, V., Khurana, J. P., Tyagi, A. K. \& Khurana, P. An update on chloroplast genomes. Plant Syst. Evol. 271, 101-122 (2007).

23. Daniell, $\mathrm{H}$. et al. The complete nucleotide sequence of the cassava (Manihot esculenta) chloroplast genome and the evolution of atpF in Malpighiales: RNA 
editing and multiple losses of a group \|I intron. Theor. Appl. Genet. 116, 723-737 (2008).

24. Saski, C. et al. Complete chloroplast genome sequence of Gycine max and comparative analyses with other legume genomes. Plant Mol. Biol. 59, 309-322 (2005).

25. Kim, K. et al. Comprehensive survey of genetic diversity in chloroplast genomes and $45 S$ nrDNAs within Panax ginseng species. PLoS One 10, e0117159 (2015).

26. Kazakoff, S. H. et al. Capturing the biofuel wellhead and powerhouse: the chloroplast and mitochondrial genomes of the leguminous feedstock tree Pongamia pinnata. PLoS One 7, e51687 (2012).

27. Ma, P. F., Zhang, Y. X., Zeng, C. X., Guo, Z. H. \& Li, D. Z. Chloroplast phylogenomic analyses resolve deep-level relationships of an intractable bamboo tribe Arundinarieae (poaceae). Syst. Biol. 63, 933-950 (2014).

28. Tangphatsornruang, $\mathrm{S}$. et al. The chloroplast genome sequence of mungbean (Vigna radiata) determined by high-throughput pyrosequencing: structural organization and phylogenetic relationships. DNA Res. 17, 11-22 (2010).

29. Katayama, H. \& Uematsu, C. Structural analysis of chloroplast DNA in Prunus (Rosaceae): evolution, genetic diversity and unequal mutations. Theor. Appl. Genet. 111, 1430-1439 (2005).

30. Yao, X. et al. Chloroplast genome structure in Ilex (Aquifoliaceae). Sci. Rep. $\mathbf{6}$, 28559 (2016).

31. Yang, J. B., Li, D. Z. \& Li, H. T. Highly effective sequencing whole chloroplast genomes of angiosperms by nine novel universal primer pairs. Mol. Ecol. Resour. 14, 1024-1031 (2014)

32. Zhang, Q. et al. The genetic relationship and structure of some natural interspecific hybrids in Prunus subgenus Prunophora, based on nuclear and chloroplast simple sequence repeats. Genet. Resour. Crop Evol. 65, 625-636 (2017).

33. Martin, G., Baurens, F. C., Cardi, C., Aury, J. M. \& D'Hont, A. The complete chloroplast genome of banana (Musa acuminata, Zingiberales): insight into plastid monocotyledon evolution. PLoS One 8, e67350 (2013).

34. Melotto-Passarin, D. M., Tambarussi, E. V., Dressano, K., De Martin, V. F. \& Carrer, H. Characterization of chloroplast DNA microsatellites from Saccharum spp and related species. Genet. Mol. Res. 10, 2024-2033 (2011).
35. Hu, Y., Woeste, K. E. \& Zhao, P. Completion of the chloroplast genomes of five Chinese Juglans and their contribution to chloroplast phylogeny. Front. Plant Sci. 7, 1955 (2016)

36. Guisinger, M. M., Kuehl, J. V., Boore, J. L. \& Jansen, R. K. Extreme reconfiguration of plastid genomes in the angiosperm family Geraniaceae: rearrangements, repeats, and codon usage. Mol. Biol. Evol. 28, 583-600 (2011).

37. Weng, M. L., Blazier, J. C., Govindu, M. \& Jansen, R. K. Reconstruction of the ancestral plastid genome in Geraniaceae reveals a correlation between genome rearrangements, repeats, and nucleotide substitution rates. Mol. Biol. Evol. 31, 645-659 (2014).

38. Yang, Y. et al. Comparative analysis of the complete chloroplast genomes of five Quercus species. Front. Plant Sci. 7, 959 (2016).

39. Park, J., Kim, Y., Nam, S., Kwon, W. \& Xi, H. The complete chloroplast genome of horned holly, llex cornuta Lindl. (Aquifoliaceae). Mitochondrial DNA Part B 4, 1275-1276 (2019).

40. Rendell, S. \& Ennos, R. Chloroplast DNA diversity of the dioecious European tree Ilex aquifolium L. (English holly). Mol. Ecol. 12, 2681-2688 (2003).

41. Chen, T. et al. Genetic diversity and population structure of Chinese Cherry revealed by chloroplast DNA trnQ-rps16 intergenic spacers variation. Genet. Resour. Crop Evol. 60, 1859-1871 (2013).

42. Francisco-Ortega, J., Goertzen, L. R., Santos-Guerra, A., Benabid, A. \& Jansen, R. K. Molecular systematics of the Asteriscus alliance (Asteraceae: Inuleae) I: evidence from the internal transcribed spacers of nuclear ribosomal DNA. Syst. Bot. 24, 249-266 (1999).

43. Jansen, R. K. et al. Analysis of 81 genes from 64 plastid genomes resolves relationships in angiosperms and identifies genome-scale evolutionary patterns. Proc. Natl Acad. Sci. USA 104, 19369-19374 (2007).

44. Joshi, A. et al. Phylogenetic relationships among low-ploidy species of Poa using chloroplast sequences. Genome 60, 384-392 (2017).

45. Lohse, M., Drechsel, O. \& Bock, R. OrganellarGenomeDRAW (OGDRAW): a tool for the easy generation of high-quality custom graphical maps of plastid and mitochondrial genomes. Curr. Genet. 52, 267-274 (2007).

46. Kurtz, S. et al. REPuter: the manifold applications of repeat analysis on a genomic scale. Nucleic Acids Res. 29, 4633-4642 (2001).

47. Katoh, K, Kuma, K, Toh, H. \& Miyata, T. MAFFT version 5: improvement in accuracy of multiple sequence alignment. Nucleic Acids Res. 33, 511-518 (2005). 